\title{
CYTOTOXIC LACTONE-TYPE DITERPENOIDS AND TRITERPENOID FROM Vitex pubescens Vahl
}

\author{
L.Anwar ${ }^{1}$, A. Santoni ${ }^{2}$, D.P.Putra ${ }^{3}$ and M. Efdi ${ }^{2, *}$ \\ ${ }^{1}$ Department of MIPA Education, Universitas Riau, Pekanbaru, Riau, Indonesia \\ ${ }^{2}$ Department of Chemistry, Andalas University, Padang, West Sumatra, Indonesia \\ ${ }^{3}$ Faculty of Pharmacy, Andalas University, Padang, West Sumatra, Indonesia \\ *E-mail: maiefdi@sci.unand.ac.id
}

\begin{abstract}
The stem bark of $V$. pubescens Vahl has been used as a traditional medicine in Indonesia. Isolation of the stem bark of $V$. pubescens Vahl with some column chromatography techniques led to obtaining the lactone diterpenoids, neoandrographolide (1), andrographolide (2), 14-deoxyandrographolide (3), and a pentacyclic triterpenoid compound, betulinic acid (4). Neoandrographolide (1) was reported in this plant for the first time. Cytotoxic activity of compounds 1-4 showed inactivity against HeLa (MTT assay).

Keywords: Cytotoxic, V. pubescens Vahl, 14-Deoxyandrographolide, Andrographolide, Neoandrographolide, Betulinic Acid.
\end{abstract}

@ $\mathrm{RAS} \bar{Y} Y A N$. All rights reserved

\section{INTRODUCTION}

Vitex genus consists of 300 species distributed in the tropics and sub tropics ${ }^{1}$. This genus is rich in organic compounds, some of which are flavonoid, terpenoid, ecdysteroid, and iridoid glucoside ${ }^{2}$. Many of them have interesting biological activities, such as anticancer ${ }^{3-5}$, antiinflammation ${ }^{6,7}$, antioxidant ${ }^{8}$, antimicrobial $^{9}$, antitrypanosomal ${ }^{10}$, antilarvaside ${ }^{11}$, and antituberculosis ${ }^{12}$.

$V$. pubescens Vahl is one of the Vitex genus which grows in the Asian territorial, such as Indonesia, Malaysia, India, Sri Lanka, and the Philippines. In Indonesia, it is mainly found in Sumatra, Kalimantan, and Java island ${ }^{1}$. V. pubescens Vahl is used for traditional treatment for many diseases, its leaves and stem bark are used for back pain, cut, indigestion, fever, scorpion sting, increase appetite, dysentery, antiinflammation, cancer and rhinitis, and also used to increase stamina ${ }^{13}$. Ecdysteroid:pinnatasterone, 20hydroxyecdisone, turkesterone ${ }^{15}$,iridoid glucoside: pinnatoside and flavonoid:viscioside, apigenin and luteolin ${ }^{16}$ have been isolated from $V$. pubescens Vahl stem bark. Meanwhile, $\beta$-sitosterol, stigmasterol, 5hydroxy-3, 7, 4'-trimethoxyflavone, 5-hydroxy-7,4'-dimethoxy-flavone, and 5-hydroxy-3,3',4',7tetramethoxyflavone ${ }^{17}$ were also found in Vitex pinnata which grows in Malaysia.

Diterpenoid is the main secondary metabolites in Vitex. Lactone-type diterpenoid which has furan ring from $\mathrm{C} 13$ to $\mathrm{C} 16$ is considered as a specific constituent in this genus ${ }^{18}$. Diterpenoid compounds have interesting and diverse biological activities, such as 6-acetoxy-9-hydroxy-13[14]-labdan-16,15-olide, vitexilactone, rotundi-furan, vitetrifolin $\mathrm{D}$, and vitetrifolin $\mathrm{E}^{19}$ which are active as anticancer agents, 9hydroxy-13(14)-labden-15,16-olide and isoambreinolide which have antituberculosis ${ }^{20}$ property and five diterpenoid compounds from Vitex trifolia, 4,4,8,10-tetramethyl-trans-decalin skeleton, vitexifolin E, vitexifolin F, vitexilactone, and 6-acetoxy-9-hydroxy-13(14)-labdan-16,15-olide which have good activity as antitripanosomal ${ }^{10}$.

As a part of our research on Indonesian herbal medicine potencial as cytotoxic agents, in this paper will be reported the isolation and characterization of neoandrographolide (1) and its activity against cervix cancer cells (HeLa). The anticancer assay was also carried out on the three other compounds (2-4).

Rasayan J. Chem., 12(3), 1641-1645 (2019)

http://dx.doi.org/10.31788/RJC.2019.1235025

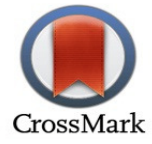


RASĀYAN J. Chem.

Vol. 12 | No. 3 |1641 - 1645| July - September | 2019

\section{Plant Material}

\section{EXPERIMENTAL}

The stem bark of $V$. pubescens Vahl was taken from the environment of the University of Riau, Pekanbaru, Indonesia. The plant was identified at the Andalas University Herbarium (ANDA), Biology Department, Andalas University.

\section{Extraction and Isolation}

$V$. pubescens Vahl stem bark $(9.58 \mathrm{~kg})$ was dried, ground, and macerated with methanol. Maceration process was carried out for three days with three repetitions. The extract was evaporated to yield methanol crude extract $(532.2 \mathrm{~g})$. The methanol extract was fractionated with $\mathrm{n}$-hexane, dichloromethane, and ethyl acetate (EtOAc) solvent, respectively to get n-hexane extract $(60.04 \mathrm{~g})$, dichloromethane extract $(57.82 \mathrm{~g})$, ethyl acetate extract $(227.79 \mathrm{~g})$, and methanol extract $(186.55 \mathrm{~g})$.

Dichloromethane extract $(57.82 \mathrm{~g})$ was separated by using vacuum liquid chromatography (VLC) with solvents of n-hexane $100 \%$, n-hexane:ethyl acetate $(20 \%, 40 \%, 60 \%, 80 \%)$ and ethyl acetate $100 \%$, to yield fraction A, B, C, D, E, and F.

Fraction E (16.278 g) was separated by column chromatography (silica 70-230 mesh) using eluent of nhexane and ethyl acetate with an increase in polarity to obtain 13 fractions (fraction $E_{1}-E_{13}$ ). Fraction $E_{11}$ $(1.125 \mathrm{mg}$ ) was purified by flash column chromatography (silica 230-400 mesh) using eluent of hexaneethyl acetate $(1: 1,4: 6,3: 7,2: 8,1: 9)$ and ethyl acetate $100 \%$ to yield 12 fractions (fraction $\mathrm{A}_{11.1}-\mathrm{A}_{11.12}$ ). Fraction $A_{11.11}$ (1.105) was purified with column chromatography (silica 230-400 mesh) with eluent DCM:EtOAc (1:1, 3:7, 0:10) to afford compound 1 (16 mg) and compound 2 (36 $\mathrm{mg})$. The isolation process of compound $\mathbf{3}$ and $\mathbf{4}$ were fully explained in previous publication ${ }^{21-23}$.

\section{Cytotoxic Activity Assay}

HeLa cells were cultured in RPMI 1640 media containing $10 \%$ fetal bovine serum (FBS) and $1 \%$ penicillin-streptomycin antibiotic and incubated in $\mathrm{CO}_{2} 5 \%$ incubator at $37^{\circ} \mathrm{C}$ for 24 hours. After 24 hours of growth, HeLa cells $\left(1 \times 10^{4}\right.$ cell/mL, $\left.100 \mu \mathrm{L}\right)$ were distributed on 96 -well plate, and $100 \mu \mathrm{L}$ of the tested compound (dissolved in DMSO) was added to the well in various concentration. The mixture of HeLa cells and the tested compound was incubated for 24 hours with $\mathrm{CO}_{2} 5 \%$ at $37^{\circ} \mathrm{C}$. After 24 hours of incubation, $10 \mu \mathrm{L}$ of MTT was added and allowed to stand for 4 hours. The living cells will react to MTT which produces a purple color of formazan. The MTT reaction was stopped with stopper reagent $(\mathrm{HCl}$ $10 \%$ in SDS), and incubated overnight at room temperature. The absorbance was then measured with ELISA reader at $\lambda 595 \mathrm{~nm}$.

\section{Spectral Data}

Neoandrographolide (1).M.p.170-171 ${ }^{\circ} \mathrm{C}$; UV (MeOH) $\lambda_{\max } \mathrm{nm}: 204$; IR $v_{\max } \mathrm{cm}^{-1}: 3369$, 2927, 2868, 1746, 1070; ${ }^{1} \mathrm{H}$ NMR (500 MHz, CD $\left.{ }_{3} \mathrm{OD}\right) \delta$ ppm: $0.71(3 \mathrm{H}, \mathrm{s}, \mathrm{H}-20) ; 1.03(3 \mathrm{H}, \mathrm{s}, \mathrm{H}-18) ; 0.96$ (1H, td, H3); $1.1(1 \mathrm{H}, \mathrm{td}, \mathrm{H}-3) ;(1.27(1 \mathrm{H}, \mathrm{dd}, \mathrm{H}-5) ; 1.78(1 \mathrm{H}, \mathrm{m}, \mathrm{H}-1), 1.94(1 \mathrm{H}, \mathrm{m}, \mathrm{H}-1), 1.66(1 \mathrm{H}, \mathrm{m}, \mathrm{H}-9) ; 1.46$ $(1 \mathrm{H}, \mathrm{m}, \mathrm{H}-2) ; 1.65(1 \mathrm{H}, \mathrm{m}, \mathrm{H}-2) ; 1.37(1 \mathrm{H}, \mathrm{m}, \mathrm{H}-6) ; 1.86(1 \mathrm{H}, \mathrm{m}, \mathrm{H}-6) ; 2.0(1 \mathrm{H}, \mathrm{m}, \mathrm{H}-7) ; 2.42(1 \mathrm{H}, \mathrm{m}$, H-7); $1.62(1 \mathrm{H}, \mathrm{m}, \mathrm{H}-11) ; 1.80(1 \mathrm{H}, \mathrm{m}, \mathrm{H}-11) ; 2.1(1 \mathrm{H}, \mathrm{m}, \mathrm{H}-12) ; 2.38(1 \mathrm{H}, \mathrm{m}, \mathrm{H}-12) ; 3.21$ (1H, d, J=9.1 Hz, H-19); 4.09 (1H, d, J=9.7 Hz, H-19); $4.82(2 \mathrm{H}, \mathrm{d}$ J=1.95, H-15); 4.63 (1H, s, H-17), 4.86 (1H, s, H17), 7.34 (1H, t, J=1.95, H-14); 3.16 (1H, H-2'); 3.24 (1H, H-5'); 3.30 (1H, H-4'); 3.32 (1H, H-3'); 3.69 (1H, H-6'); 3.85 (1H, H-6'); 4.18 (1H, H-1'); ${ }^{13} \mathrm{C}_{\mathrm{NMR}}\left(125 \mathrm{MHz}, \mathrm{CD}_{3} \mathrm{OD}\right) \delta$ ppm: 177.09 (C-16), 149.42 (C-8), 147.71 (C-14), 134.91 (C-13), 107.36 (C-17), 105.16 (C-1'), 78.33 (C-3'), 77.78 (C-5'), 75.36 (C-2'), 73.54 (C-19), 72.17 (C-15), 71.78 (C-4'), 62.84 (C-6'), 57.94 (C-9), 57.78 (C-5), 40.74 (C4), 40.31 (C-1), 39.77 (C-7), 39.47 (C-10), 37.28 (C-3), 28.41 (C-18), 25.72 (C-6), 25.54 (C-12), 23.05 (C-11), 20.15 (C-2), and 15.93 (C-20). HMQC and HMBC: see Table-1.

\section{RESULTS AND DISCUSSION}

Extraction of dried stem bark of $V$. pubescens Vahl with methanol produced crude extract $(532.2 \mathrm{~g})$. It was then fractionated with n-hexane, dichloromethane, and ethyl acetate solvent, respectively. Dichloromethane fraction (57 g) was separated with VLC to yield 6 main fractions (A-F). Purification of 
RASĀYAN J. Chem.

Vol. 12 | No. 3 |1641 - 1645| July - September | 2019

fraction A, C, and E produced compound 1-4 which were identified as neoandrographolide, andrographolide, 14-deoxyandrographolide, and betulinic acid. Structure determination of compound 2-4 has been described in the previous studies ${ }^{21-22}$.

Compound 1 forms a white solid with a melting point of $170-171^{\circ} \mathrm{C}$. The IR spectra showed absorption for hydroxyl group, carbonyl group, and C-H aliphatic at $v_{\text {maks }} 3369,1746$ and $2927 \mathrm{~cm}-1$, respectively.1H NMR (Table 1) spectrum indicated several characteristics of the Compound 1. There was the presence of a vinyl group $(\delta \mathrm{H} 4.63$ and $4.86 \mathrm{ppm}, \mathrm{H}-17)$, and two oxygenated methylenes $(\delta \mathrm{H} 4.82$ ppm, H-15; 3.21, and 4.09 ppm, H-19). Two olefin bonds at C-14 and C-17 provided chemical shift at $\delta \mathrm{H} 4.63-7.34 \mathrm{ppm}$. The proton at $\mathrm{C}-14$ showed at higher frequency $(\delta \mathrm{H} 7.34 \mathrm{ppm})$ due to conjugation effect of the carbonyl group. 1H-NMR spectrum exhibited some signals suitable for glucosil group, were identified as one oxidized methylene carbon $\left(\delta \mathrm{H} 3.69\right.$ and $\left.3.85 \mathrm{ppm}, \mathrm{H}-6^{\prime}\right)$, five oxidized methinecarbones at $\delta \mathrm{H}^{2} 4.18 \mathrm{ppm}\left(\mathrm{H}_{-1} 1^{\prime}\right), 3.16 \mathrm{ppm}\left(\mathrm{H}-2^{\prime}\right), 3.32 \mathrm{ppm}\left(\mathrm{H}-3^{\prime}\right), 3.30 \mathrm{ppm}\left(\mathrm{H}^{\prime} 4^{\prime}\right)$, and 3.24 ppm (H-5'). 13C NMR and DEPT data analysis (Table 1) revealed some signals for 26 carbon atoms. Two methyl carbons displayed at $\delta \mathrm{C} 15.93 \mathrm{ppm}(\mathrm{C}-20)$ and $28.41 \mathrm{ppm}(\mathrm{C}-18)$. The presence of glucosyl group was marked by several signals at $\delta \mathrm{C} 105.16 \mathrm{ppm}\left(\mathrm{H}^{-1}{ }^{\prime}\right), 75.36\left(\mathrm{H}-2^{\prime}\right), 78.33$ (H-3'), 71.78 (H-4'), $77.83 \mathrm{ppm}\left(\mathrm{H}-5^{\prime}\right)$, and $62.48 \mathrm{ppm}(\mathrm{H}-6$ '). Moreover, there were also found the signals for C-carbonyl $(\delta$ $177.06 \mathrm{ppm}, \mathrm{C}-16)$, two oxidized methylene carbons $(\delta 72.17, \mathrm{C}-15$ and $73.54 \mathrm{ppm}, \mathrm{C}-19)$. The presence of olefin methine carbons was marked by signals at $\delta 147.71 \mathrm{ppm}(\mathrm{C}-14)$ and one hybridized methylene carbon sp2 at $107.36 \mathrm{ppm}$ (C-17). Spectra analysis of 1H-1H COSY, HMQC, and HMBC supported compound 1 as neoandrographolide. The data of compound 1 was confirmed by the previous research ${ }^{23}$.

Table-1: ${ }^{1} \mathrm{H}$ and ${ }^{13} \mathrm{C}$ NMR Data of Compound 1

\begin{tabular}{|c|c|c|c|c|c|}
\hline $\begin{array}{l}\text { No } \\
\text { C }\end{array}$ & $\begin{array}{c}\delta_{\mathrm{H}}(\mathrm{ppm}), \text { integrity, } \\
\text { multiplicity, } J\end{array}$ & $\begin{array}{c}\delta_{\mathrm{C}} \\
(\mathrm{ppm})\end{array}$ & DEPT & $\begin{array}{c}\delta_{\mathrm{C}} \\
(\mathrm{ppm}) *\end{array}$ & HMBC \\
\hline 1 & $1,94(\mathrm{~m}) ; 1,78(\mathrm{~m})$ & 40,31 & $\mathrm{CH}_{2}$ & 40,23 & $\mathrm{C} 4, \mathrm{C} 6 \mathrm{C} 8, \mathrm{C} 9$ \\
\hline 2 & $1,65(\mathrm{~m}) ; 1,46(\mathrm{~m})$ & 20,15 & $\mathrm{CH}_{2}$ & 20,26 & $\mathrm{C} 1, \mathrm{C} 4$ \\
\hline 3 & $\begin{array}{c}1,1(\mathrm{td}, \mathrm{J}=3,25 ; 13 \mathrm{~Hz})) ; 0,96 \\
(\mathrm{td}, \mathrm{J}=3,9 ; 13,6 \mathrm{~Hz})\end{array}$ & 37,28 & $\mathrm{CH}_{2}$ & 37,21 & $\mathrm{C} 2, \mathrm{C} 5, \mathrm{C} 18, \mathrm{C} 19$ \\
\hline 4 & - & 40,74 & $\mathrm{C}$ & 40,66 & \\
\hline 5 & $1,27(\mathrm{dd}, \mathrm{J}=2 ; 13 \mathrm{~Hz})$ & 57,78 & $\mathrm{CH}$ & 57,71 & $\mathrm{C} 6, \mathrm{C} 9, \mathrm{C} 10, \mathrm{C} 18, \mathrm{C} 19, \mathrm{C} 20$ \\
\hline 6 & $1,86(\mathrm{dt}) ; 1,37(\mathrm{td})$ & 25,72 & $\mathrm{CH}_{2}$ & 25,44 & $\mathrm{C} 5, \mathrm{C} 7, \mathrm{C} 8, \mathrm{C} 10$ \\
\hline 7 & $2,42(\mathrm{~m}) ; 2(\mathrm{~m})$ & 39,77 & $\mathrm{CH}_{2}$ & 39,68 & $\mathrm{C} 5, \mathrm{C} 6, \mathrm{C} 8, \mathrm{C} 17$ \\
\hline 8 & - & 149,32 & $\mathrm{C}$ & 127,2 & \\
\hline 9 & $1,66(\mathrm{~m})$ & 57,94 & $\mathrm{CH}$ & 57,86 & $\mathrm{C} 4, \mathrm{C} 5, \mathrm{C} 8, \mathrm{C} 11, \mathrm{C} 12, \mathrm{C} 17, \mathrm{C} 20$ \\
\hline 10 & - & 39,47 & $\mathrm{C}$ & 39,37 & \\
\hline 11 & $1,8(\mathrm{~m}) ; 1,62(\mathrm{~m})$ & 23,05 & $\mathrm{CH}_{2}$ & 22,97 & $\mathrm{C} 8, \mathrm{C} 9, \mathrm{C} 12, \mathrm{C} 17, \mathrm{C} 20$ \\
\hline 12 & $2,38(\mathrm{~m}) ; 2,1(\mathrm{~m})$ & 25,54 & $\mathrm{CH}_{2}$ & 25,64 & $\mathrm{C} 10, \mathrm{C} 11, \mathrm{C} 13, \mathrm{C} 14, \mathrm{C} 16$ \\
\hline 13 & - & 134,91 & $\mathrm{C}$ & 134,83 & \\
\hline 14 & $7,34(\mathrm{t}, \mathrm{J}=1,95 \mathrm{~Hz})$ & 147,71 & $\mathrm{CH}$ & 147,59 & $\mathrm{C} 12, \mathrm{C} 13, \mathrm{C} 15, \mathrm{C} 16$ \\
\hline 15 & $4,82(\mathrm{~d}, \mathrm{~J}=1,95 \mathrm{~Hz})$ & 72,17 & $\mathrm{CH}_{2}$ & 72,06 & $\mathrm{C} 13, \mathrm{C} 14, \mathrm{C} 16$ \\
\hline 16 & - & 177,09 & $\mathrm{C}$ & 176,89 & \\
\hline 17 & $4,86(\mathrm{~s}) ; 4,63(\mathrm{~s})$ & 107,36 & $\mathrm{CH}_{2}$ & 107,25 & $\mathrm{C} 7, \mathrm{C} 8, \mathrm{C} 9$ \\
\hline 18 & $1,03(\mathrm{~s})$ & 28,41 & $\mathrm{CH}_{3}$ & 28,31 & $\mathrm{C} 2, \mathrm{C} 3, \mathrm{C} 5, \mathrm{C} 10, \mathrm{C} 19$ \\
\hline 19 & $\begin{array}{c}4,09(\mathrm{~d}, \mathrm{~J}=9,7 \mathrm{~Hz}) ; 3,21 \\
(\mathrm{~d}=9,1 \mathrm{~Hz})\end{array}$ & 73,54 & $\mathrm{CH}_{2}$ & 73,46 & $\mathrm{C} 3, \mathrm{C} 10, \mathrm{C} 18, \mathrm{C} 1{ }^{\prime}$ \\
\hline 20 & $0,71(\mathrm{~s})$ & 15,93 & $\mathrm{CH}_{3}$ & 15,84 & $\mathrm{C} 1, \mathrm{C} 5$ \\
\hline $1^{\prime}$ & $4,18(\mathrm{~d}, 7,8 \mathrm{~Hz}))$ & 105,16 & $\mathrm{CH}$ & 105,07 & C19 \\
\hline 2 ' & $3,16(\mathrm{t}, \mathrm{J}=9,1 \mathrm{~Hz})$ & 75,36 & $\mathrm{CH}$ & 75,28 & C1', C3', C4', \\
\hline 3 & $3,32(\mathrm{t}, \mathrm{J}=8 \mathrm{~Hz})$ & 78,33 & $\mathrm{CH}$ & 78,25 & C4', \\
\hline $4{ }^{\prime}$ & $3,30(\mathrm{t}, \mathrm{J}=9,05 \mathrm{~Hz})$ & 71,78 & $\mathrm{CH}$ & 71,72 & C6', C5' \\
\hline 5 & $3,24(\mathrm{~m})$ & 77,78 & $\mathrm{CH}$ & 77,74 & $4 '$ \\
\hline 6 ' & $\begin{array}{c}3,85(\mathrm{dd}, \mathrm{J}=2 ; 11,7 \mathrm{~Hz}) \\
3,69(\mathrm{dd}, \mathrm{J}=5,2 ; 11,7 \mathrm{~Hz})\end{array}$ & 62,84 & $\mathrm{CH}_{2}$ & 62,77 & $\mathrm{C}^{\prime}, \mathrm{C} 5{ }^{\prime}$ \\
\hline
\end{tabular}


Study of the literature showed neoandrographolide and 14-deoxyandrographolide were found for the first time in Vitex genus. Andrographolide was previously found in V. limonifolia ${ }^{24}$, and betulinic acid was also isolated from $V$. trifolia ${ }^{25}$ and $V$. negundo ${ }^{26}$.<smiles>C=C1CCC2C(C)(C)CCCC2(C)C1CCC1=CCOC1=O</smiles>

(1)<smiles>C=C1CCC2C(C)(C)C(O)CCC2(C)C1CC=C1C(=O)OCC1O</smiles>

(2)<smiles>C=C(C)C1CCC2(C(=O)O)CCC3(C)C(CCC4C5(C)CC[C@H](O)C(C)(C)C5CCC43C)C12</smiles>

(3)

(4)

Cytotoxic activity against cervix cancer cells (HeLa) was treated to 1-4. The percentage viability of isolated compounds at 25 and $50 \mu \mathrm{g} / \mathrm{mL}$ is displayed at Picture 1. Andrographolide (2) gave good inhibition where the viability of HeLa cells reached $72.7 \%$ at $50 \mu \mathrm{g} / \mathrm{mL}$. Compound 1,3 , and 4 had no effect at this concentration. $\mathrm{IC}_{50}$ value showed no potential activity of the tested compounds due to the low value of $\mathrm{IC}_{50}\left(\mathrm{IC}_{50}<30 \mu \mathrm{g} / \mathrm{mL}\right)^{27}$.

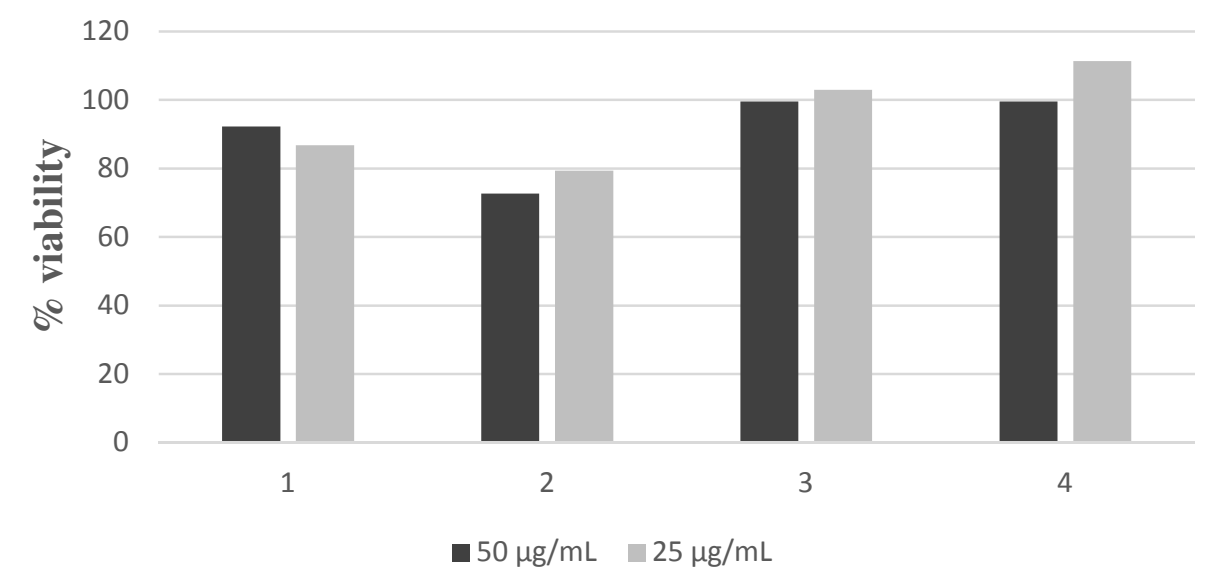

Fig.-1: Antiproliferative effects of isolated compounds of $V$. pubescens Vahl stem barks on cervix cancer cells (HeLa)

\section{CONCLUSION}

Three lactone diterpenoid compounds, neoandrographolide (1), andrographolide (2), 14deoxyandrographolide (3), and a triterpenoid compound, betulinic acid (4), had been isolated from the stem bark of $V$. pubescens Vahl. Compound 1-4 showed no antiproliferative effect on HeLa cells with $\mathrm{IC}_{50}>100 \mu \mathrm{g} / \mathrm{mL}$.

\section{ACKNOWLEDGMENT}

We would like to thank Directorate General of Higher Education for the grant through BPPDN scholarship and Doctoral Dissertation Research (no. 270//UN.19.5.1.3/PP/2018). Also thank to Andalas University Herbarium (ANDA) for identifying the specimen, The Sumatera Biota Laboratory and LIPI Serpong for taking the spectra of isolated compounds.

\section{REFERENCES}

1. R. De Kok, Kew. Bull., 63, 17(2008), DOI: 10.1007/s12225-007-9013-7

2. S. Ganapaty and K.N. Vidyadhar, J Nat Remedies,5(2), 75(2005), DOI: 10.18311/jnr/2005/391 
RASĀYAN J. Chem.

Vol. 12 | No. 3 |1641 - 1645| July - September | 2019

3. G. Seelinger, I. Merfort,U. Wolfle, and C.M. Schempp, Molecules, 13, 2628(2008), DOI: 10.3390/molecules13102628

4. H. Kim, J. Yi, N.S. Kim, Y.J. Lee, J. Kim, D. Oh, S. Oh, O. Bang, J. Lee, J Korean Soc Appl Biol Chem., 55, 433 (2012), DOI: 10.1007/s13765-012-2027-3

5. Y. Deng, Y. Chin, H. Chai, E.C. de Blanco, L.B.S. Kardono, S. Riswan, D.D. Soejarto, N.R. Farnsworth, A.D. Kinghorn, Phytochem Lett., 4, 213(2011), DOI: 10.1016/j.phytol.2011.03.007

6. T. Rastogi, M. Kubde, I.A. Farooqui, S.S. Khadabadi, Int J PharmSci Res.,1(9), 23(2010).

7. M. Li, X. Su, J. Sun, Y. Gu, Z. Huang, K. Zeng, Q. Zhang, Y. Zhao, D. Ferreira, J.K. Zjawiony, J. Li, P. Tu, J Nat Prod.,77(10), 2248 (2014), DOI:10.1021/np500509q

8. N. Tiwari, S. Luqman, N. Masood, M.M. Gupta, J Pharm Biomed Anal.,61, 207(2012), DOI: 10.1016/j.jpba.2011.12.007

9. G. Keerti and K. Padma, Int J Drug Dev Res., 4, 4 (2012).

10. F. Kiuchi, K. Matsuo, M. Ito, T.K. Qui, G. Honda, Chem Pharm Bull.,52(12), 1492(2004), DOI: $10.1248 / \mathrm{cpb} .52 .1492$

11. A. Kannathasan, A. Senthilkumar, V. Venkatesalu, Acta Trop., 120, 115(2011), DOI: 10.1016/j.actatropica.2011.07.001

12. N. Tiwari, J. Thakur,D. Saikia, M.M. Gupta, Phytomedicine, 20, 605 (2013), DOI: 10.1016/j.phymed.2013.01.003

13. K. Heyne,Tumbuhan Berguna Indonesia III. Badan Penelitian dan Pengembangan Kehutanan. Departemen Kehutanan, Jakarta, (1987).

14. A.K. Meena, U.S. Niranjan, M.M. Rao, M.M. Padhi, R. Babu, Asian J. Trad. Med., 6(2), 54(2011).

15. A. Suksamrarn, C. Sommecchai, Phytochemistry,32(2),303(1993), DOI:10.1016/S00319422(00)94985-9

16. A. Ata, N. Mbong, C.D. Iverson, R. Samarasekera, Nat Prod Commun., 4(1),1(2009).

17. N. Kamal, C.Clements, A.I. Gray, R.E.Ebel, Journal of Applied Pharmaceutical Science 6(1), 102 (2016), DOI: $10.7324 / J A P S .2016 .600117$

18. C.Z. Li, Y.F. Su, X.I Ji, Chin Tradit Herbal Drugs, 36, 930 (2005).

19. W.X. Li, C.B. Cui, B. Cai, X.S. Yao, J Asian Nat Prod Res.,7(2),95(2005), DOI: $10.1080 / 10286020310001617165$

20. N. Tiwari, J. Thakur, D. Saikia, M.M. Gupta, Phytomedicine, 20,605(2013), DOI: 10.1016/j.phymed.2013.01.003

21. L.Anwar; S. Ibrahim, D.P.Putra, M. Efdi, J Chem Pharm Res.,7(10),290(2015).

22. L. Anwar,M. Efdi, M. Ninomiya, S. Ibrahim, D.P. Putra, K. Tanaka, M. Koketsu,Med Chem Res., 26(10), 2357(2017), DOI: 10.1007/s00044-017-1937-3

23. M.D. Al-Amin, M.D.M. Islam, M.M.A. Siddiqi, M.M. Haque, S. Akter, S. Ahmed, N. Sultana, and A.M.S. Chowdhury, Dhaka Univ. J. Sci.,60(1),1(2012), DOI: 10.3329/dujs.v60i1.10326

24. S. Aphaijitt, K. Nimgirawath, A. Suksamrarn, U. Tooptakong, Australian Journal of Chemistry, 48(1), 133(2006), DOI: 10.1071/CH9950133

25. M. Huang, L.Zhong, J.Xie, F. Wang, Y. Zhang, Helvetica Chimica Acta, 96(11), 2040(2013), DOI: 10.1002/hlca.201200614

26. C. Zheng, B. Huang, Y. Wu, T. Han, Q. Zhang, H. Zhang, L. Qin, Biochemical Systematics and Ecology, 38, 247(2010), DOI: 10.1016/j.bse.2010.01.002

27. S. Cao, V.H.L. Sng, X. Wu, K. Sim, B.H.K. Tan, J.T. Pereira, and S.H. Goh, Tetrahedron,54, 10915(1998), DOI: 10.1016/S0040-4020(98)00644-9

[RJC-5025/2019] 